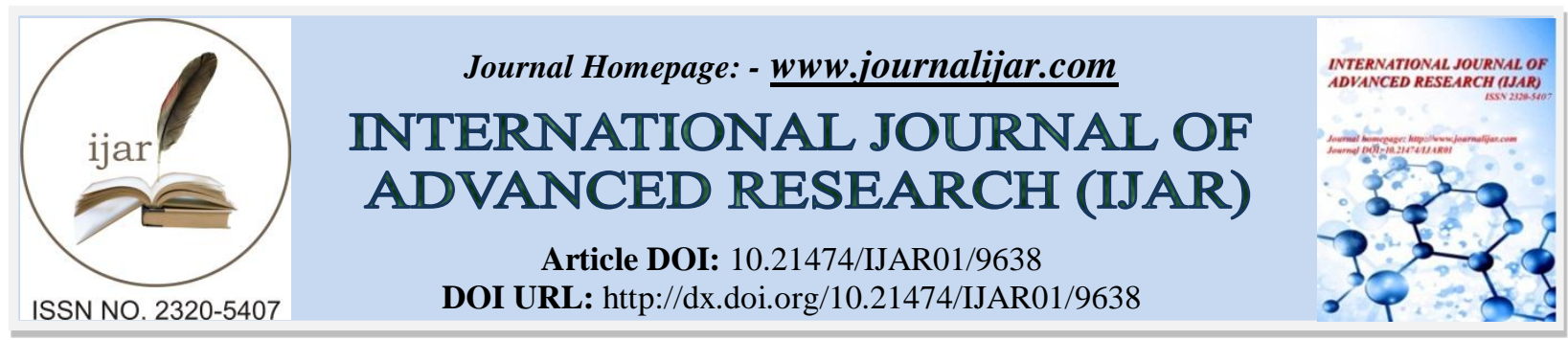

RESEARCH ARTICLE

\title{
ETUDE CINETIQUE DE LA PHOTODEGRADATION DU COLORANT « RED 6 » EN SOLUTION AQUEUSE.
}

\section{Moussa Diarra $^{1}$, Soro Donafologo Baba ${ }^{2}$, David Léonce Kouadio ${ }^{1}$, Aboua Narcisse Kouassi ${ }^{2}$, Yao Jaurès Kouassi $^{1}$, Valéry Paul Djamatchè Akesse ${ }^{1}$, Mamadou Koné ${ }^{2}$, Bini Kouamé Dongui ${ }^{1}$, Ardjouma Dembélée And Karim Sory Traore ${ }^{2,3}$.}

1. Université Jean Lorougnon GUEDE, Unité de Formation et de Recherche Environnement, Laboratoire des Sciences et Technologie de l'Environnement, BP 150 Daloa, Côte d'Ivoire.

2. Laboratoire des sciences de l'environnement, UFR des Sciences et gestion de l'environnement, Université Nangui ABROGOUA, 02 B.P 801 Abidjan 02, Côte d'Ivoire.

3. Laboratoire Central d'agrochimie et d'Ecotoxicologie, LANADA, 04 BP 612 Abidjan 04.

\section{Manuscript Info}

Manuscript History

Received: 02 July 2019

Final Accepted: 04 August 2019

Published: September 2019

Key words:

Photolysis, photocatalysis, Red 6 dye, titanium dioxide.

\begin{abstract}
The dyes are widely consumed as an input in artisan dyeing in Ivory Coast. Their detection in surface water and in groundwater is evidence of the lack of training in the good practice of textile dyeing and the environmental pollution that results from it. The dye "Red 6" widely used in dye compositions, was selected for this study. With the aim of eliminating them in synthetic waste simulating wastewater from dyeing plants, direct photolysis and photocatalysis were carried out respectively in a cylindrical reactor placed on a magnetic stirrer and 12 $\mathrm{cm}$ below an axial cap containing the irradiation lamp. The experiments were carried out with a polychromatic $(\lambda \geq 285 \mathrm{~nm})$ and monochromatic $(\lambda=254 \mathrm{~nm})$ lamp. The results indicate that the molecule is difficult to degrade with degradation rates of $24.49 \%$ and $38.77 \%$ respectively for the lamps used. The use of titanium dioxide $\left(\mathrm{TiO}_{2}\right)$ as a catalyst allowed a better degradation of the "Red 6" dye under the polychromatic lamp compared to direct photolysis with a degradation rate of $98.18 \%$ after 300 min of irradiation
\end{abstract}

Copy Right, IJAR, 2019,. All rights reserved.

\section{Introduction}

Ces dernières décennies, la pollution de l'eau est devenue une préoccupation environnementale des politiques, des sociétés et même du monde scientifique. En effet, la présence et le devenir des colorants dans l'environnement, même à de faibles concentrations, suscitent des inquiétudes au regard du grand nombre de publications et d'écrits sur ces composés chimiques dits «polluants émergents » (Hammami, 2008). Selon (Trabelsi, 2014), la plupart des colorants sont récalcitrants du fait de leur très faible biodégradabilité, leur persistance dans l'environnement. (Guo et al. 2015).

En Côte d'Ivoire, la situation est beaucoup plus préoccupante du fait de l'inexistence des systèmes d'épuration des effluents dans les teintureries artisanales et du mauvais état de fonctionnement des réseaux de collecte des eaux usées (Ministère des eaux et forêts, 2008; Dalogo, 2015). Les effluents issus des teintureries sont déversés à l'état brut dans les égouts, les ruelles et les canalisations pour aboutir à la pollution des eaux de surface et souterraine.

Corresponding Author:-Moussa Diarra.

Address:-Université Jean Lorougnon GUEDE, Unité de Formation et de Recherche Environnement, 124 
(Dalogo, 2015). Face à cette problématique, il convient de prévenir le danger en proposant des voies et méthodes d'élimination de ces polluants avant leur rejet dans les milieux récepteurs. A cet effet, de nombreuses études se sont consacrées ces vingt (20) dernières années à l'émergence de nouveaux procédés de traitement des polluants organiques et minéraux parmi lesquels l'adsorption (Khalfaoui, 2012 ; Yeddou et al., 2012 ; Koffi, 2013) et les procédés d'oxydation avancée (PAO) (Kouadio, 2011; Lemlikchi, 2012 ; Soro, 2012; Diarra, 2013 ; Kouakou, 2014).

Cependant, hormis les travaux de Dalogo (2015), les voies d'expérimentation de ces techniques dans le traitement des eaux résiduaires de teinture ont été moins explorées en Côte d'Ivoire. C'est dans ce contexte que notre étude se propose d'étudier la dégradation par voie photochimique du colorant «Red 6 » en solution aqueuse. C'est une molécule beaucoup utilisée dans de nombreux secteurs (chimie, pharmacologie, médecine, biologie, textile...). L'utilisation ignorante de cette substance engendre des conséquences graves sur la santé humaine et sur l'environnement.

\section{Matériel et Méthodes}

\section{Réactifs et solvants}

1. L'eau déionisée utilisée est fournie par un système Milli-Q (Millipore)

2. Le dioxyde de titane "Degussa P-25": Sa surface spécifique est de $54 \mathrm{~m}^{2} \cdot \mathrm{g}^{-1}$ et sa structure cristalline est constituée de $98 \%$ d'anatase et $2 \%$ de rutile. $\mathrm{Le}^{\mathrm{TiO}} 2$ provient de la société prolabochemicals (France)

3. Le colorant «Red 6 » de pureté $99 \%$ et utilisé dans toutes les techniques analytiques pour les préparations des solutions provient de la société PanreacQuimicaSau (Espagne). Ses propriétés physico-chimiques sont regroupées dans le Tableau I

Tableau I: Caractéristiques physico-chimiques du «Red 6

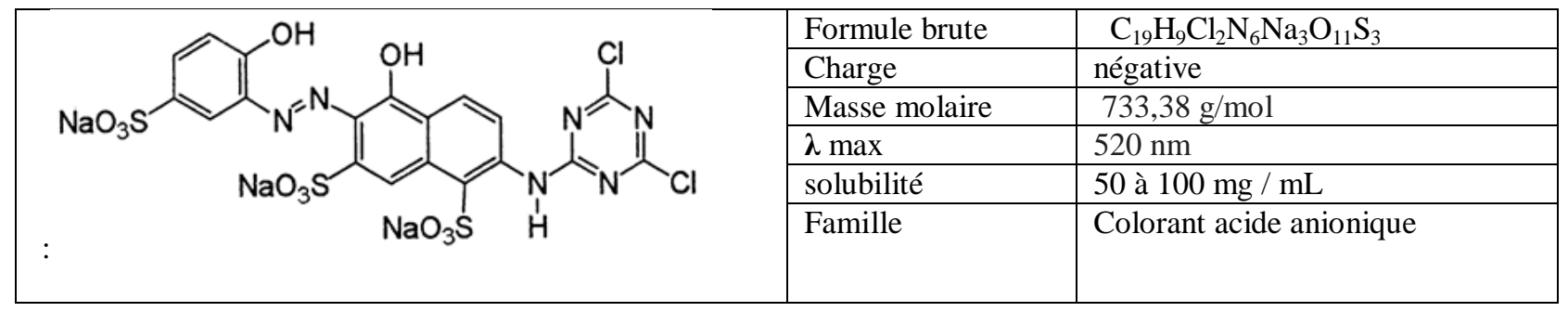

\section{Dispositif d'irradiation}

Le dispositif d'irradiation utilisé est une rampe équipée d'une source lumineuse sous laquelle un réacteur de $500 \mathrm{~mL}$ en pyrex est disposé. La lampe est positionnée horizontalement à $12 \mathrm{~cm}$ au-dessus du réacteur. L'irradiation a été faite soit avec une lampe monochromatique $\mathrm{T} 6 \mathrm{C}$ de marque Heraeus (NN 40/20) à vapeur de mercure basse pression ,de puissance $40 \mathrm{~W}$ et de spectre d'emission $254 \mathrm{~nm}$, soit avec une lampe polychromatique Vilbert et Loumart T6M de puissance de $6 \mathrm{~W} / \mathrm{cm}^{2}$ et dont le spectre d'émission s'étale de $285 \mathrm{~nm}$ à $500 \mathrm{~nm}$. L'agitation de la suspension est réalisée par un agitateur magnétique supportant le réacteur. L'ensemble du dispositif est entouré d'un film plastique noir pendant l'expérience pour isoler le réacteur de la lumière.

\section{Protocole expérimental}

\section{Préparation de la solution à irradier}

Le « Red 6 » est un produit très soluble dans l'eau. Pour sa mise en solution aqueuse, $0,5 \mathrm{~g}$ du produit sont mis dans une fiole bouchée de 1 litre contenant de l'eau ultrapure préalablement chauffée à $75^{\circ} \mathrm{C}$. La fiole contenant la solution préparée $\left(681,7 \mu \mathrm{mol} . \mathrm{L}^{-1}\right)$ est entourée de papier aluminium et conservée à l'abri de la lumière.

\section{Pour la photolyse directe}

Des prélèvements d'échantillons ont été effectués à l'aide de pipette pasteur à intervalles de $30 \mathrm{mn}$ durant $3 \mathrm{~h}$ dans un premier temps et dans un second temps par intervalle d'une heure (1h) pendant deux heures $(2 \mathrm{~h})$. Ces échantillons étiquetés sont conservés au frais à l'abri de la lumière solaire. 


\section{Pour la photocatalyse}

Les expériences de photocatalyse ont été effectuées avec $500 \mathrm{~mL}$ de solution contenant $6 \mu \mathrm{mol} . \mathrm{L}^{-1}$ du colorant « Red 6 » et 0,2 g. $\mathrm{L}^{-1}$ de dioxyde de titane $\left(\mathrm{TiO}_{2}\right)$ mis en suspension. La suspension a été agitée pendant 30 min à l'obscurité afin d'atteindre l'équilibre d'adsorption entre la molécule et la surface du catalyseur avant l'irradiation avec la lampe polychromatique. Pendant des intervalles de temps définis, des échantillons-ont été prélevés, filtrés avec un filtre à seringue de $0,45 \mu \mathrm{m}$ avant leur analyse. L'influence des paramètres tels que la concentration en catalyseur et le $\mathrm{pH}$ de la solution du colorant a été mis en évidence.

\section{Influence du pH}

Pour mettre en évidence l'influence du $\mathrm{pH}$ sur la photo-dégradation du « Red 6 » en présence de $\mathrm{TiO}_{2}$, nous avons choisis trois solutions de $\mathrm{pH}$ 6, 7 et 9. Ces valeurs sont ajustées par addition de $\mathrm{HCl}(1 \mathrm{~N})$ ou $\mathrm{NaOH}(1 \mathrm{~N})$, tout en gardant les concentrations, la température ambiante et la vitesse d'agitation constantes.

\section{Méthodes Analytiques}

\section{Etude cinétique de la photolyse}

La cinétique de décoloration a été déterminée en utilisant la spectrophotométrie d'absorption moléculaire à la longueur d'onde $520 \mathrm{~nm}$ qui est dans la partie visible du spectre électromagnétique. La cinétique de dégradation du «Red $6 »$ a été déterminée en utilisant les mesures des absorbances à la longueur d'onde $520 \mathrm{~nm}$.

\section{pH-métrie}

Les mesures de $\mathrm{pH}$ ont été effectuées à l'aide d'un pH-mètre HANNA muni d'une électrode préalablement étalonné. L'étalonnage de l'appareil a été effectué de façon journalière en deux points avec des tampons à pH 7,1 et pH 4,1.

\section{Résultats et Discussion}

\section{Aspect cinétique de la dégradation du colorant: Influence de la source lumineuse.}

La figure 1 montre les cinétiques de dégradation respectivement sous la lampe monochromatique $(\lambda=254 \mathrm{~nm})$ et sous la lampe polychromatique $(\lambda \geq 285 \mathrm{~nm})$.

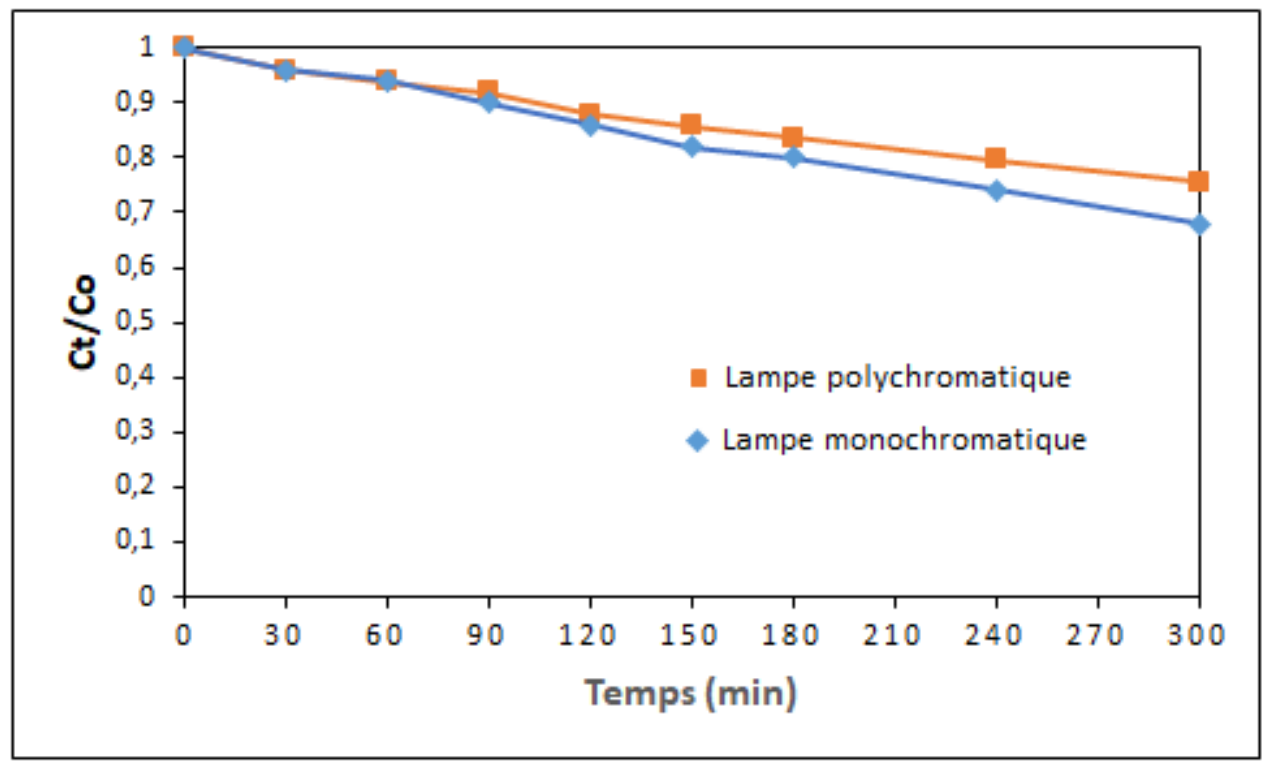

Figure 1:-Cinétique de la photolyse directe du colorant « $\operatorname{Red} 6 »$ à $\lambda=254 \mathrm{~nm}$ et à $\lambda \geq 285 \mathrm{~nm}$.

Elle montre que le colorant se dégrade difficilement quel que soit la lampe utilisée. Toutefois, le processus de dégradation à $\lambda \geq 285 \mathrm{~nm}$ pour nos conditions expérimentales a été beaucoup plus lent que celui obtenu à $\lambda=254$ $\mathrm{nm}$. Les pourcentages de dégradation obtenus après 300 minutes d'irradiation, à $\lambda \geq 285 \mathrm{~nm}$ et $254 \mathrm{~nm}$ sont respectivement de $24,49 \%$ et $38,77 \%$. Ces résultats sont semblables avec ceux obtenus par Chenini (2007) au cours de l'étude de la dégradation par voie photochimique du colorant « Orange $\mathrm{G}$ » en solution aqueuse. Ces études ont montré que seulement $9,1 \%$ de la concentration initiale du colorant «Orange $G$ » était éliminé après 5 heures (300minutes) d'irradiation avec la lampe monochromatique $(\lambda=254 \mathrm{~nm})$ contre $5 \%$ avec la lampe polychromatique 
$(\lambda \geq 285 \mathrm{~nm})$. Ce fait pourrait être attribué au flux photonique incident émis par cette source monochromatique, plus énergétique comparativement à celui émis à $\lambda \geq 285 \mathrm{~nm}$.

La figure 2 rapporte les représentations semi-logarithmiques construites à partir des cinétiques de dégradation sous les deux lampes monochromatique $(\lambda=254 \mathrm{~nm})$ et polychromatique $(\lambda \geq 285 \mathrm{~nm})$.

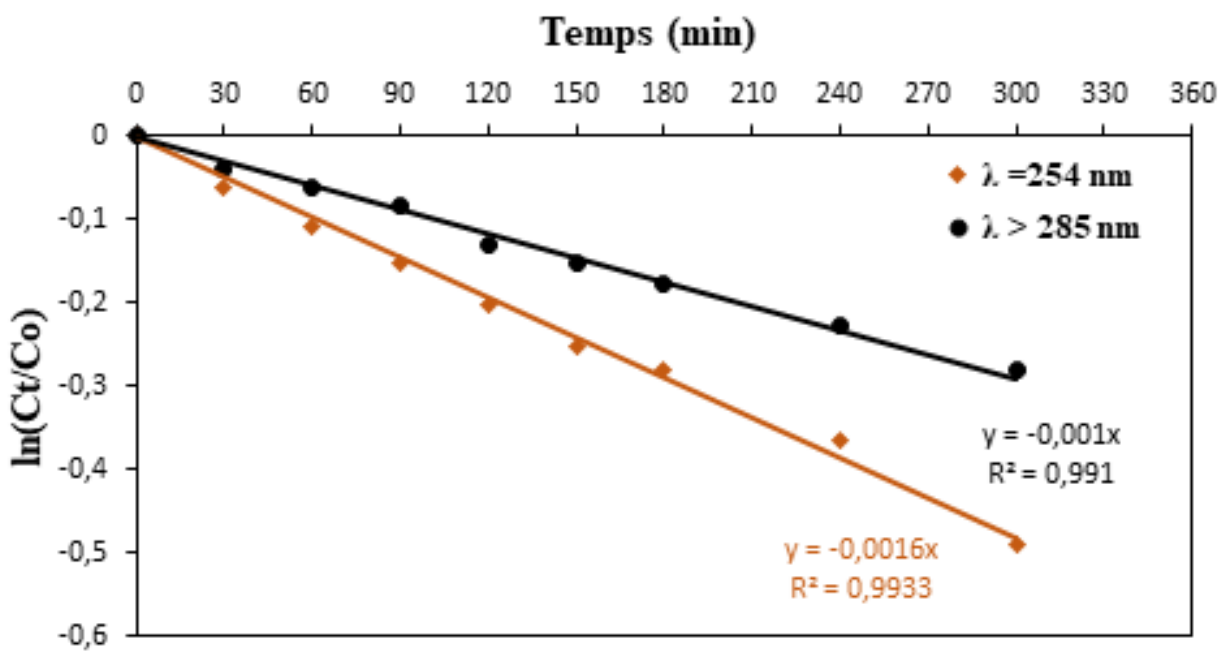

Figure 2:-Courbe semi-logarithmique de la photolyse directe du colorant « Red 6 » à $\lambda=254 \mathrm{~nm}$ et à $\lambda \geq 285 \mathrm{~nm}$.

Elle montre que la vitesse de photolyse du colorant «Red 6 » respectivement à $\lambda=254 \mathrm{~nm}$ et à $\lambda \geq 285 \mathrm{~nm}$ obéit à une loi cinétique d'ordre un apparent.

\section{Photolyse du colorant « Red 6 » en présence d'un catalyseur $\left(\mathrm{TiO}_{2}\right)$}

La réaction de photo- catalyse du colorant « Red 6 » $\left(6 \mu\right.$ mol.L-1) a été réalisée en milieu aqueux $\left(\mathrm{pH}=6,33 ; 25^{\circ} \mathrm{C}\right)$ avec une lampe polychromatique $(\lambda \geq 285 \mathrm{~nm})$. La solution à irradier est obtenue par dispersion de $\mathrm{TiO}_{2}$ de concentration $0,2 \mathrm{mg} . \mathrm{L}^{-1}$ dans la solution « Red $6 »$.

La figure 3 présente la cinétique obtenues par le système $\mathrm{UV} / \mathrm{TiO}_{2}$ (photocatalyse) du colorant « Red 6 » sous la lampe polychromatique.

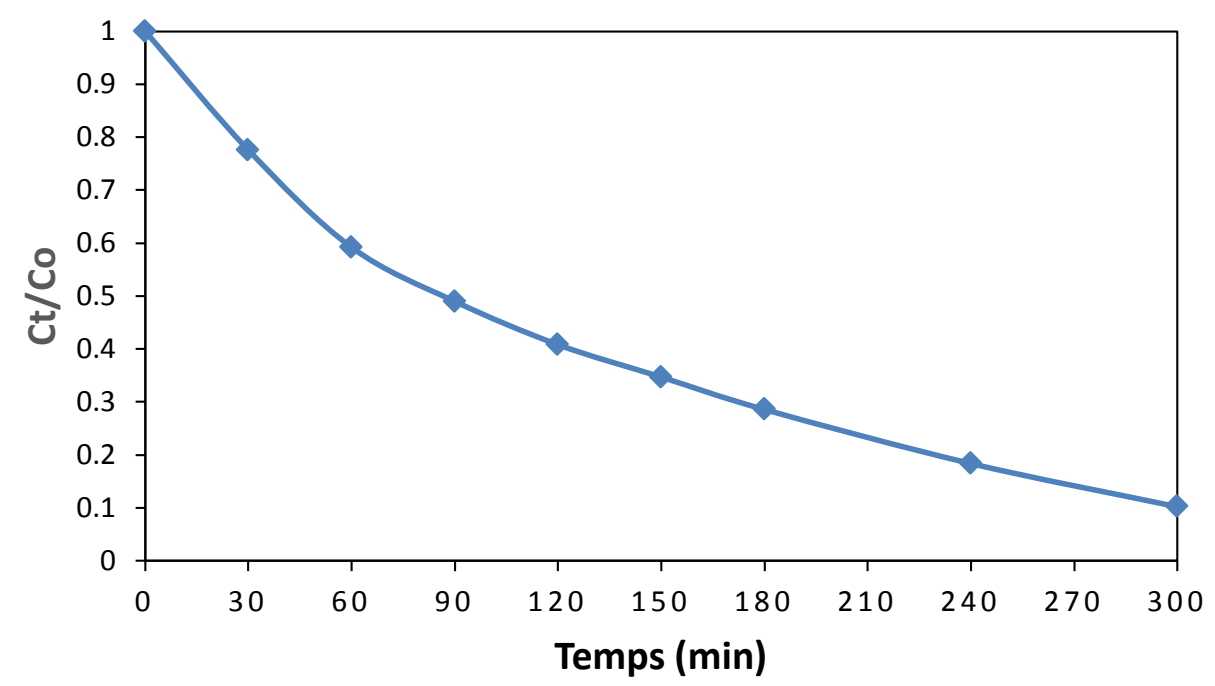

Figure 3:-Cinétique de la photocatalyse du colorant « Red $6 »\left(6 \mu\right.$ mol. $\left.\mathrm{L}^{-1}\right)$ en milieu aqueux $\left(\mathrm{pH}=6,33 ; 25^{\circ} \mathrm{C}\right)$ sous lampe polychromatique 
Après 300 min d'irradiation, le taux de dégradation du produit initial a été de $98,18 \%$ en présence du $\mathrm{TiO}_{2}$.

Quant à la figure 4, elle présente des exemples de cinétiques obtenues par le système UV (photolyse directe) d'une part et d'autre part par le système $\mathrm{UV} / \mathrm{TiO}_{2}$ (photocatalyse) du colorant « Red 6 » sous la lampe polychromatique

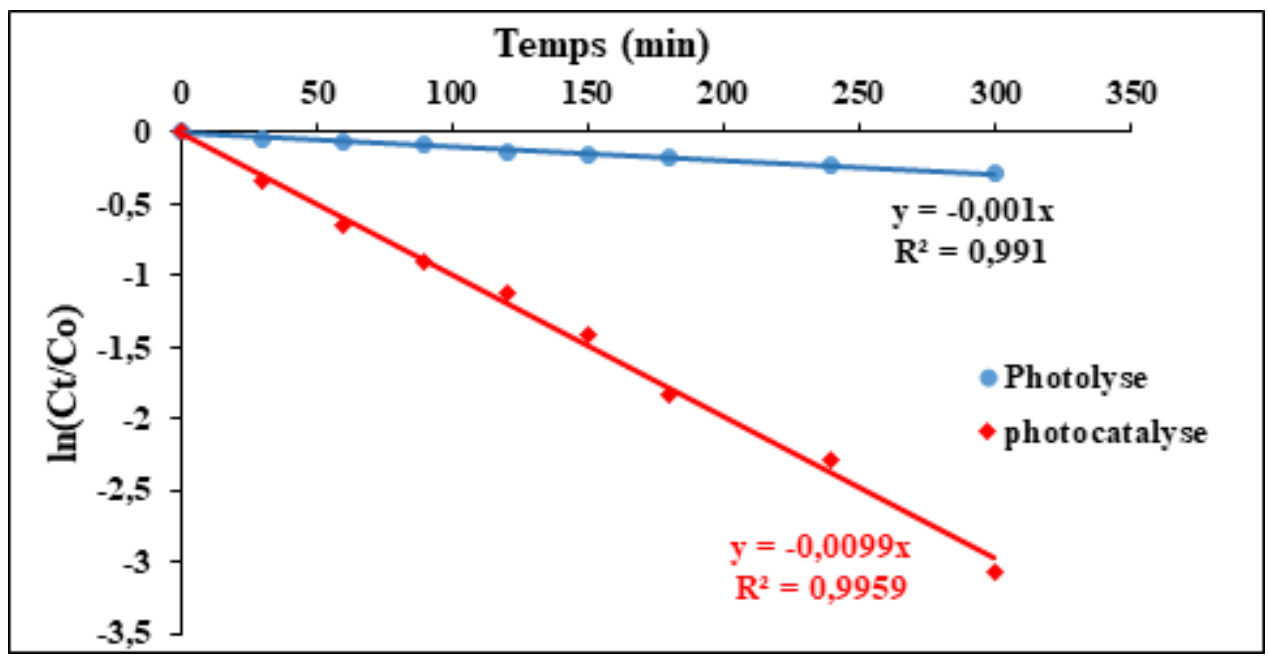

Figure 4:-Comparaison des cinétiques de photolyse et de la photocatalyse du « Red 6 » en solution aqueuse.

Les constantes de vitesse $(\mathrm{k})$ d'ordre un apparent et les temps de demi-vie $\left(\mathrm{t}_{1 / 2}\right)$, son rapportés dans le tableau 2.

Tableau 2:-Paramètres cinétiques de la dégradation du colorant «Red 6 »: ordre de réaction

\begin{tabular}{|c|c|c|c|}
\hline Expérience & $\mathrm{R}^{2}$ & $\mathrm{k}\left(\mathrm{min}^{-1}\right)$ & $\mathrm{t}_{1 / 2}(\mathrm{~min})$ \\
\hline Photolyse à $\lambda \geq 285 \mathrm{~nm}$ & 0,9910 & 0,0010 & 693,1472 \\
\hline Photocatalyse à $\lambda \geq 285 \mathrm{~nm}$ & 0,9959 & 0,0099 & 70,01487 \\
\hline
\end{tabular}

La cinétique par la photolyse catalysée du colorant « Red 6 » avec l'emploi du $\mathrm{TiO}_{2}$ est environ 9 fois plus rapide que celle de la photolyse directe en absence du $\mathrm{TiO}_{2}$. En effet, l'absorption de radiation UV par le semi-conducteur $\mathrm{TiO}_{2}$ crée une déficience en électron, créant des trous $\left(\mathrm{h}^{+}\right)$dans la bande de valence $(\mathrm{BV})$ et libère des électrons libres (e) dans la bande de conduction (BC). Ces électrons migrant à la surface du semi-conducteur $\mathrm{TiO}_{2}$ peuvent réduire l'oxygène dissous et former des radicaux anions superoxydes $\mathrm{O}_{2}{ }^{\bullet-}$. Ceux-ci peuvent réagir avec $\mathrm{H}_{2} \mathrm{O}$ pour donner du peroxyde $d^{\prime}$ 'hydrogène conduisant à la formation des espèces très réactives $\left(\mathrm{OH}^{\bullet}\right)$. Ces radicaux très réactifs, reconnus comme premiers agents oxydants, vont ainsi attaquer et dégrader le substrat organique en solution suivant les équations:

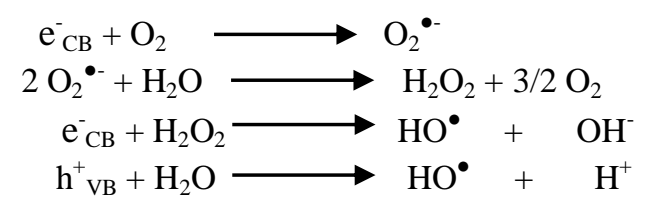

\section{Influence du pH sur la photocatalyse du colorant « Red 6 »}

Le $\mathrm{pH}$ de la solution aqueuse affecte énormément la charge de surface du $\mathrm{TiO}_{2}$, Le $\mathrm{pH}$ pour lequel la charge de surface d'un oxyde est nulle, est appelé Point de Zéro Charge $\left(\mathrm{pH}_{\mathrm{PZC}}\right)$, il vaut 6,5 pour le $\mathrm{TiO}_{2}($ Bouzaida et als, 2004). Pour des valeurs différentes de ce $\mathrm{pH}$, la surface de l'oxyde est chargée. En effet, si le pH est supérieur au $\mathrm{pH}_{\mathrm{PZC}}$ du $\mathrm{TiO}_{2}$, la surface du photo-catalyseur est chargée négativement. Elle est de charge positive pour une valeur de $\mathrm{pH}$ inférieure à $\mathrm{pH}_{\mathrm{PZC}}$. Ceci pourrait être expliqué par les réactions de surface photocatalytique présentées cidessous

$$
\begin{array}{cc}
\mathrm{TiOH}+\mathrm{H}^{+} \rightarrow \mathrm{TiOH}_{2}^{+} & \text {pour } p H<p H_{p z c}=6,5 \\
\mathrm{TiOH}+\mathrm{OH}^{-} \rightarrow \mathrm{TiO}^{-}+\mathrm{H}_{2} \mathrm{O} & \text { pour } p H>p H_{p z c}=6,5
\end{array}
$$


Les résultats représentés sur la Figure 5 montrent que l'efficacité catalytique augmente avec la diminution du pH. La dégradation maximale du «Red 6 » est observée à $\mathrm{pH}=6$ avec un taux de degradation de 98,18\%. L'effet du pH initial de la solution sur la photo-décomposition est très important car il influe sur la charge électrique de surface du catalyseur. En effet, en milieu acide, une forte adsorption du colorant sur la surface de l'oxyde est observée, celle-ci est, probablement, due à l'attraction électrostatique de la charge positive du $\mathrm{TiO}_{2}$ et la charge négative du colorant. Cependant, la vitesse de la photo-dégradation diminue avec l'augmentation du $\mathrm{pH}$.

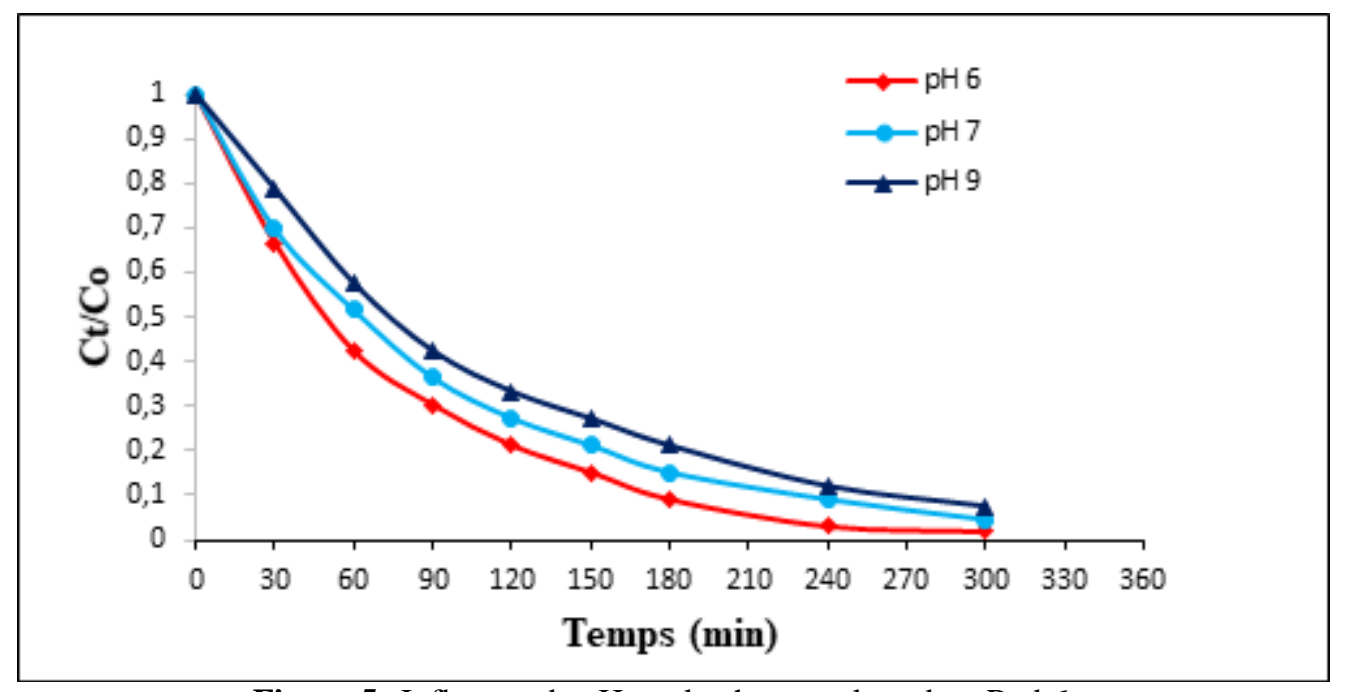

Figure 5:-Influence de $\mathrm{pH}$ sur la photocatalyse du « Red $6 »$

\section{Conclusion:-}

Cette étude, qui s'inscrit dans le cadre général de la dépollution des effluents contenant des colorants, a eu pour objectif d'étudier la photodégradation du colorant « Red 6 » contenue dans les effluents de teinture. Elle a permis de comparer les performances des procédés de différents systèmes photochimiques (systèmes UV seul, et $\mathrm{UV} / \mathrm{TiO}_{2}$,) utilisés pour la minéralisation de polluants organiques. Les résultats expérimentaux ont mis en évidence que les effluents colorés peuvent être réhabilité par des processus photochimiques avant leur rejet dans l'environnement ou être affectés à d'autres usages. Le colorant « Red 6 » se dégrade très lentement par irradiation UV à $254 \mathrm{~nm}$ comme par irradiation polychromatique à $\lambda \geq 285 \mathrm{~nm}$ avec un taux de dégradation respectif de $38,77 \%$ et $24,49 \%$ après 300 minutes d'irradiation. L'étude de la photocatalyse a montré l'effet du catalyseur sur la dégradation de colorant « red 6 ». Le taux de dégradation en présence du $\mathrm{TiO}_{2}$ a été de 98,18\%. La dégradation de ce colorant, quel que soit le système d'oxydation expérimenté dans nos conditions de travail, suit une cinétique du premier ordre. Ces résultats ont permis de montrer que le procédé utilisant le système $\mathrm{UV} / \mathrm{TiO}_{2}$ donne des rendements plus satisfaisants dans les processus de dégradation abiotiques naturels du colorant « Red $6 »$.

\section{References:-}

1. Alahiane S., Qourzal S., El Ouardi M., Belmouden M., Assabbane A. \& Ait-Ichou Y. (2013).

2. Adsorption and photocatalytic degradation of indigo carmine dye in aqueous solutions using $\left(\mathrm{TiO}_{2} / \mathrm{UV}^{-\mathrm{O}_{2}}\right)$. Journal of Materials and Environmental Science, 4(2): pp: 239-250.

3. Bouzaida I., Ferronato C., Chovelon J.M., Rammah M.E. \& Hermann J.M. (2004).Heterogeneous photocatalitic degradation of the anthraquinone dye, Acide Blue 2(AB25) a kenetic approach. Journal of Photochemistry and Photobiology A: Chemistry, 168: pp: 23 -30.

4. Chenini H. (2007). Etude de la degradation par voie photochimique de polluant organiques « orange $G$ » en milieu aqueux homogene et heterogene. Thèse de Doctorat. Université Mentouri Constantine, Algérie, $176 \mathrm{p}$.

5. Dalogo K.A.P. (2015). Caractérisation et décontamination des eaux contenant les colorants parles procédés d'adsorption et d'oxydation avancee: cas des effluents de teinture artisanale dans le district d'Abidjan. Thèse de doctorat, Université Nangui Abrogoua, Côte d'Ivoire, 190 p.

6. Guo J., Chen X., Shi Y., Lan Y. \& Qin C. (2015). Rapid Photodegradation of Methyl Orange (MO) assisted with $\mathrm{Cu}$ (II) and tartaric acid. Plos one 10 (8): doi: 10.1371. 
7. Hammami S. (2008). Étude de dégradation des colorants de textile par les procédés d'oxydation avancée. Application à la dépollution des rejets industriels». Thèse du doctorat, Université Paris-Est et Tunis El Manar, $209 \mathrm{p}$.

8. Khalfaoui A.D. (2012). Etude expérimentale de l'élimination de polluants organiques et inorganiques par adsorption sur des matériaux naturels: application aux peaux d'orange et de banane. Thèse de Doctorat, Université Mentouri. Constantine, Algérie, 143 p.

9. Koffi A.L.C. (2013). Etude des propriétés de biosorption de la sciure de Triplochiton scleroxylon: Application au traitement d'effluents métalliques synthétiques. Thèse de Doctorat, Université Nangui Abrogoua- Institut National Polytechnique Houphouët-Boigny, Côte d'Ivoire, 155 p.

10. Kouadio D.L. (2011). Contamination des eaux par quelques antibiotiques et antiinflammatoires non-stéroïdiens et traitement photochimique: Cas de la commune de Yopougon. Thèse de doctorat, Université d'Abobo Adjamé, Côte d'Ivoire, $152 \mathrm{p}$.

11. Kouakou K. (2014). Réhabilitation des effluents phytosanitaires issus du rinçage des pulvérisateurs: Cas de quatre molécules de la famille des urées substituées (le chlorotoluron, l'isoproturon, le linuron et le métobromuron). Thèse de doctorat, Université d'Abobo Adjamé, Cote d'Ivoire, $118 \mathrm{p}$.

12. Lemlikchi W. (2012). Elimination de la pollution des eaux industrielles par différents procèdes d'oxydation et de Co-précipitation. Thèse de doctorat, Université Mouloud Tizi-Ouzou, 177p.

13. Ministère des Eaux et Forêts, (2003). Gestion intégrée des ressources en eau en Côte d'Ivoire: Bilan et Perspectives, République de Côte d'Ivoire, $67 \mathrm{p}$.

14. Soro D.B. (2012). Photodégradation en milieu aqueux de trois pesticides: thiabendazole, diméthoate et diazinon. Thèse de Doctorat, Université d'Abobo Adjamé (Abidjan, Cote d'Ivoire), $172 \mathrm{p}$.

15. Trabelsi H. (2014). Etude de la dégradabilité et de la toxicité des colorants par ozonation et photocatalyse. Thèse de doctorat, Université de Monastir, Tunisie, $159 \mathrm{p}$.

16. Yeddou M.N., Bensaadi Z., Lagha H. \& Bensmaili A. (2012). Etude de l'adsorption d'une mixture de composés biorécalcitrants en milieu aqueux. Larhyss Journal, 11: pp: 7-16. 